\title{
REABILITAÇÃO DAS FUNÇÕES DO OLFATO E DO PALADAR EM LARINGECTOMIZADOS TOTAIS: REVISÃO SISTEMÁTICA
}

\author{
Rehabilitation of smell and taste functions \\ in total laryngectomy: Systematic Review
}

\author{
Ada Salvetti Cavalcanti Caldas ${ }^{(1)}$, Vera Lúcia Dutra Facundes ${ }^{(2)}$, Hilton Justino da Silva ${ }^{(3)}$
}

\begin{abstract}
RESUMO
Tema: na laringectomia total ocorre a transferência do fluxo aéreo nasal definitivamente ao traqueostoma, podendo provocar diminuição na percepção do olfato e do paladar. Recentemente tem sido desenvolvidos métodos de intervenção a fim de melhorar a performance olfativa e gustativa em laringectomizados, embora sejam escassos os estudos que abordam esse tema. Objetivo: rever de forma sistemática as evidências de como as técnicas envolvidas na reabilitação das funções do olfato e do paladar tem efeito em indivíduos laringectomizados totais. Conclusão: nesta revisão todos os estudos selecionados demonstraram eficácia na utilização de técnicas de reabilitação para a função do olfato em laringectomizados totais. Para o paladar não houve propostas para intervir diretamente nesta função.
\end{abstract}

DESCRITORES: Laringectomia; Olfato; Paladar; Transtornos do Olfato; Distúrbios do Paladar; Reabilitação

\section{INTRODUÇÃO}

A laringectomia total é um tratamento cirúrgico em que há remoção de estruturas que produzem o som laríngeo e de músculos vizinhos, com a transferência do fluxo aéreo nasal definitivamente ao traqueostoma ${ }^{1}$, comprometendo a chegada de moléculas odoríferas ao epitélio olfativo por inalação nasal, provocando diminuição na percepção do olfato e consequentemente do paladar ${ }^{2,3}$.

Recentemente tem sido desenvolvidos métodos de intervenção a fim de melhorar a performance olfativa e gustativa em laringectomizados,

(1) Terapeuta Ocupacional; Mestranda em Patologia pela Universidade Federal de Pernambuco, UFPE, Recife, PE Brasil; Especialista em Saúde Pública pela Universidade de Pernambuco - UPE.

(2) Terapeuta Ocupacional; Professora adjunto do curso de Terapia Ocupacional da Universidade Federal de Pernambuco, UFPE, Recife, PE - Brasil; Doutora em Neuropsiquiatria pela Universidade Federal de Pernambuco - UFPE.

(3) Fonoaudiólogo; Professor adjunto II da Universidade Federal de Pernambuco - UFPE, Recife, PE - Brasil; Doutor em Nutrição pela Universidade Federal de Pernambuco UFPE.

Conflito de interesses: inexistente destacando-se técnicas de reabilitação que proporcionem um aumento do fluxo aéreo nasal com a melhora na chegada de moléculas odoríferas ao neuroepitélio olfativo ${ }^{4,5}$.

Tendo em vista os poucos estudos que abordam esse tema, o presente artigo tem como objetivo revisar de forma sistemática as evidências de como as técnicas envolvidas na reabilitação das funções do olfato e do paladar tem efeito em indivíduos laringectomizados totais.

\section{MÉTODO}

A revisão sistemática da literatura foi realizada a partir das bases de dados PUBMED, MEDLINE (1997 - 2010), MEDLINE OLD (1966 - 1996), LILACS e SciELO, tendo a busca de dados ocorrido em outubro/2010.

Para a pesquisa foram utilizados descritores (DESCs) - palavras-chaves para recuperação de assuntos da literatura científica - e termos livres (TL) - termos não encontrados no DESC e MESH, mas de relevância para a pesquisa -, sendo realizados os seguintes cruzamentos: "laryngectomy" (DESCs) AND "smell" (DESCs); "laryngectomy" 
(DESCs) AND "taste" (DESCs); "laryngectomy" (DESCs) AND "olfaction disorders" (DESCs); "laryngectomy" (DESCs) AND "taste disorders" (DESCs); "laryngectomized" (TL) AND "smell" (DESCs); "laryngectomized" (TL) AND "taste" (DESCs), e seus equivalentes em português e espanhol.

A busca foi realizada por três pesquisadores de forma independente, seguindo os critérios de inclusão e exclusão.

Como critérios de inclusão foram selecionados artigos originais (excluindo-se editoriais e estudo de caso) que abordassem a laringectomia total e a reabilitação das funções do olfato e/ou paladar nesta população com a utilização de técnicas específicas, tendo os manuscritos sido publicados nos idiomas português, inglês e espanhol.
Os artigos de revisão da literatura foram excluídos, bem como aqueles que não propuseram especificamente intervir nas funções do olfato e/ou paladar e os que não abordaram a população de laringectomizados totais, tais como estudos realizados em animais.

\section{REVISÃO DA LITERATURA}

Foram encontrados 457 artigos a partir da busca de descritores e termo livre, sendo 183 da PUBMED, 153 da MEDLINE, 109 da MEDLINE OLD, sete da LILACS, três da SCIELO Brasil e dois da SCIELO Colômbia. Considerando os critérios de inclusão e de exclusão foram selecionados 11 artigos para esta revisão, conforme aponta a Figura 1.

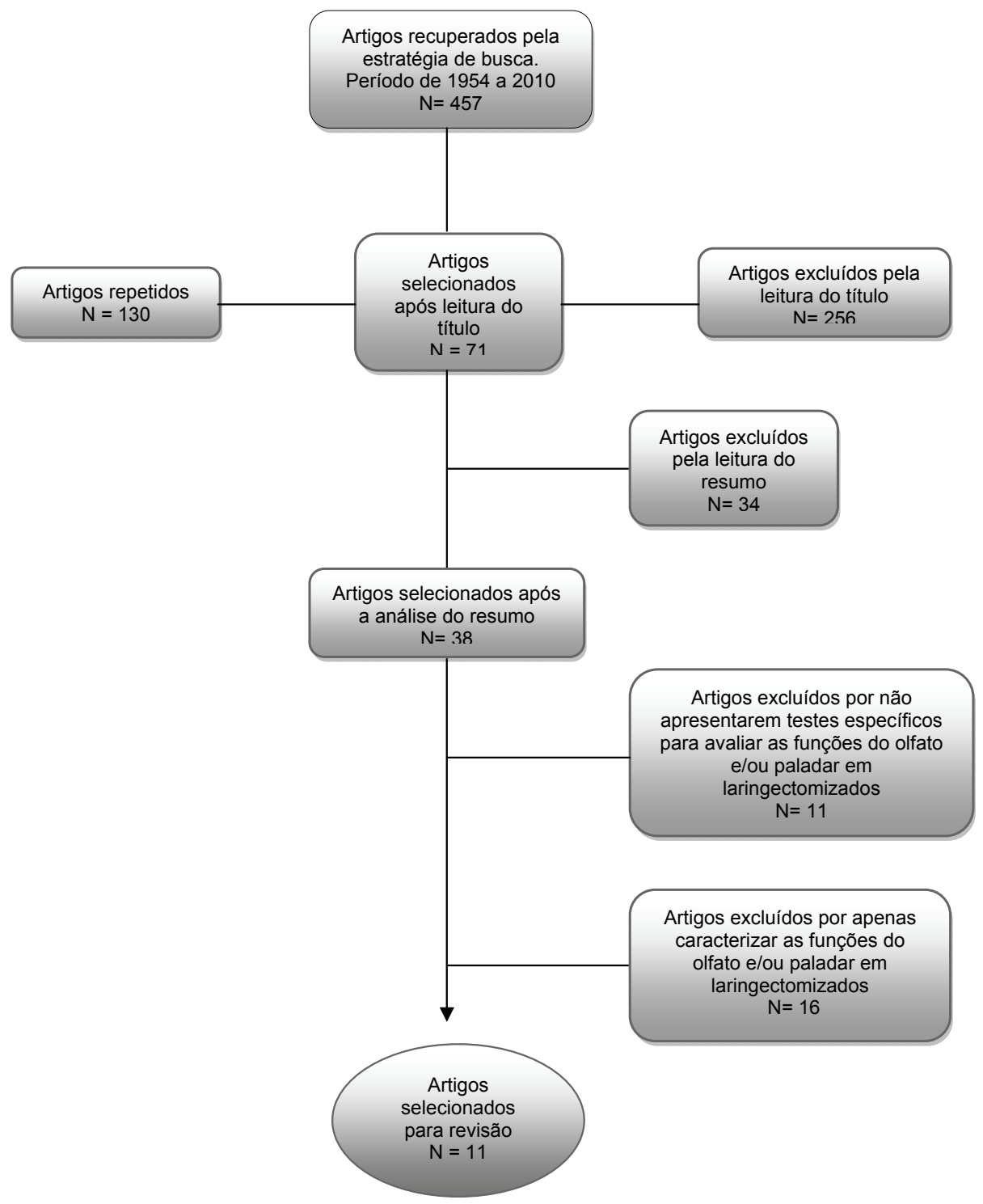

Figura 1 - Fluxograma do número de artigos encontrados e selecionados após aplicação dos critérios de inclusão e exclusão segundo descritores e bases de dado 
A grande diversidade observada nos estudos encontrados não permitiu análise estatística (metanálise). A heterogeneidade pode ser percebida com relação ausência de critérios de randomização, diversificação da amostragem e das variáveis consideradas na população de cada artigo analisado. Assim para melhor apresentação dos resultados optou-se por considerar as seguintes variáveis dos artigos selecionados: autor, ano, país, amostra, média de idade, tempo após laringectomia, teste utilizado para avaliar as funções do olfato e/ou paladar, técnica de intervenção e resultados, como podem ser observados na Tabela 1.

A reabilitação das funções do olfato e do paladar parece ter sido evidenciada em pesquisas a partir dos anos 2000. Embora o conhecimento de que a laringectomia total possa provocar diminuição na acuidade olfativa e gustativa tenha sido descrito em estudos desde $1954^{6}$, apenas no ano de $1987^{7}$ houve a primeira publicação descrevendo uma técnica de intervenção com a tentativa de reverter essas perdas sensoriais.

Acredita-se que este fato pode estar relacionado a tradicionalmente ser dada maior ênfase à reabilitação da voz ${ }^{4}$, já que tais alterações são mais evidentes nesta população, sendo pouco percebidas as implicações nas funções do olfato e consequentemente do paladar.

A descoberta da diminuição da função olfativa e do paladar em laringectomizados totais, bem como os possíveis mecanismos e estruturas envolvidas nessas alterações, parece ter estimulado grupos de pesquisadores a investigar estratégias de intervenção para melhoria dessas funções, principalmente na Holanda, Suécia e Alemanha 5,8-13.

Supõe-se que o crescimento de casos de câncer de laringe e do número de cirurgias de laringectomias totais na Europa, tem levado estudiosos cada vez mais a investigarem essa população ${ }^{8}$. Associado a este fato, pesquisas tem utilizado questionários e testes específicos para avaliar as funções do olfato e paladar com o fim de atestar a eficácia de técnicas de intervenção, constatando-se nessas publicações uma preocupação maior com a qualidade de vida desses indivíduos.

Outro ponto relevante é a ausência de estudos na América Latina, sugerindo pouca ênfase em pesquisar este tema. No Brasil estudos que abordam a qualidade de vida e a comunicação oral são a preferência de pesquisadores nesta região 14,15 , embora tenha sido encontrado um artigo de pesquisadores brasileiros fora das estratégias de busca abrangendo os critérios de inclusão e exclusão adotados desta revisão ${ }^{16}$.

A diversidade das publicações apresentadas nesta revisão pode ser evidenciada pelo número de amostragem, tendo revelado a ocorrência de estudos entre 12 e 44 indivíduos, embora tenha identificado-se uma prevalente variação entre 18 e 24 sujeitos. Supõe-se que o número reduzido de indivíduos pesquisados pode comprometer a inferência desses achados para a população geral.

Nos artigos selecionados, a idade dos sujeitos analisados ficou em torno dos 60 anos, o que está em consonância com estudos que apontam a faixa etária de 40 a 70 como a de maior prevalência para O câncer de laringe ${ }^{17}$.

Outro ponto que deve ser levado em conta na análise dos resultados aqui apresentados é o tempo de cirurgia, tendo esse tempo variado de 0 a 20 anos, reforçando assim a heterogeneidade dos estudos selecionados.

Os testes utilizados para avaliação da função olfativa em sua maioria preocuparam-se em fazer uma análise quantitativa com uso de instrumentos padronizados, o que sugere uma preocupação com os resultados apresentados, já que tem como finalidade comprovar a eficácia de uma técnica de intervenção.

Em estudo realizado com 44 indivíduos utilizouse, como método para avaliação do olfato, o Odor Detection Test (ODT) com apresentação de 16 ensaios com frascos de $250 \mathrm{~mL}$ contendo solvente inodoro de dipropileno glicol e os outros contendo dipropileno glicol com feniletanol ${ }^{13}$. Mais tarde, em outra publicação de mesma autoria, também utilizou-se este método avaliativo em conjunto com o Smell Disk Test (SDT) que consiste na apresentação de 8 diferentes odores em disquetes, tendo o estudo comprovado que SDT representou ser um teste mais rápido e de simples aplicação e reprodutibilidade ${ }^{12}$.

O Sniffin Sticks test battery é outro teste padronizado utilizado em publicações recentes ${ }^{3,5,9}$, tendo como método avaliativo a utilização de odores contidos em 12 "canetas", sendo um teste objetivo, representando maior confiança nos resultados apresentados.

Testes subjetivos com aplicação de questionários tem sido amplamente utilizados para avaliar as funções do olfato e do paladar, principalmente com relação a autoavaliação e a satisfação com a situação atual vivenciada pelo indivíduo. O questionário Olfaction, Taste and Appetite (QOTA) representa um teste subjetivo com a proposta de estudar através de questões múltiplas-escolhas a situação atual e prévia a cirurgia de laringectomia total $8,10,11,13$. Entende-se que embora este questionário tenha escores bem definidos requer que sua utilização seja acompanhada por testes objetivos para melhor apuração dos resultados. 
Tabela 1 - Estudos que analisaram as evidências das técnicas de reabilitação das funções do olfato e do paladar em laringectomizados totais

\begin{tabular}{|c|c|c|c|c|c|c|c|}
\hline Autor/ Ano & País & Amostra & $\begin{array}{l}\text { Média de } \\
\text { idade em } \\
\text { anos }\end{array}$ & $\begin{array}{l}\text { Tempo após } \\
\text { laringectomia }\end{array}$ & $\begin{array}{l}\text { Teste utilizado } \\
\text { para avaliar as } \\
\text { funções do olfato } \\
\text { e/ou paladar }\end{array}$ & $\begin{array}{l}\text { Técnica de } \\
\text { intervenção }\end{array}$ & Resultados \\
\hline $\begin{array}{l}\text { MORALES- } \\
\text { PUEBLA et al, } \\
2010^{4}\end{array}$ & Espanha & $\begin{array}{l}41 \text { (02 } \\
\text { mulheres e } \\
39 \text { homens) }\end{array}$ & 65,04 & 4 anos (média) & $\begin{array}{l}\text { Teste da acuidade } \\
\text { olfatória; } \\
\text { Entrevista } \\
\text { Semi-estruturada; } \\
\text { Uso de LB; }\end{array}$ & NAIM & $\begin{array}{l}\text { Antes de aprender a técnica: } \\
21,95 \%(n=9) \text { capazes de detectar } \\
\text { odores; } \\
\text { Na autoavaliação identificaram } \\
\text { a perda de } 53 \% \text { na capacidade } \\
\text { gustativa e } 97,5 \% \text { na capacidade } \\
\text { olfativa após a cirurgia. Após a } \\
\text { reabilitação classificaram de } 69 \% \\
\text { e } 44 \% \text { a perda gustativa e olfativa } \\
\text { respectivamente. }\end{array}$ \\
\hline
\end{tabular}

Nos testes, após a utilização da técnica, 90,24\% ( $\mathrm{n}=37)$ eram capazes de identificar odores; $88 \%$ dos que tinham anosmia tiveram resultado positivo com a técnica;

\begin{tabular}{|c|c|c|c|c|c|c|c|}
\hline $\begin{array}{l}\text { WARD et al, } \\
2010^{3}\end{array}$ & Austrália & $\begin{array}{l}43(06 \\
\text { mulheres e } \\
37 \text { homens) }\end{array}$ & 63,6 & $\begin{array}{l}\text { Pelo menos } 6 \\
\text { meses }\end{array}$ & SStb; & 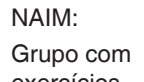 & $\begin{array}{l}\text { Antes do treino à Em domicílio: } \\
65 \% \text { anosmia; } 35 \% \text { hiposmia; } \\
\text { Na clínica: } 85 \% \text { anosmia; } 15 \%\end{array}$ \\
\hline
\end{tabular}

em domicílio; hiposmia;

Grupo com Após treino com NAIMà Em

assistência domicílio: 35\% anosmia;

clínica; $\quad 30 \%$ hiposmia; $10 \%$ normal;

Assistência na clínica: $20 \%$ anosmia; $55 \%$ hiposmia; $20 \%$ normal;

Após 3 meses de treino à Em domicílio: 20\% anosmia; 35\% hiposmia; $20 \%$ normal; Assistência na clínica: $25 \%$ anosmia; $50 \%$ hiposmia; $20 \%$ normal;

Treino na clínica provoca maior melhora na acuidade olfativa;

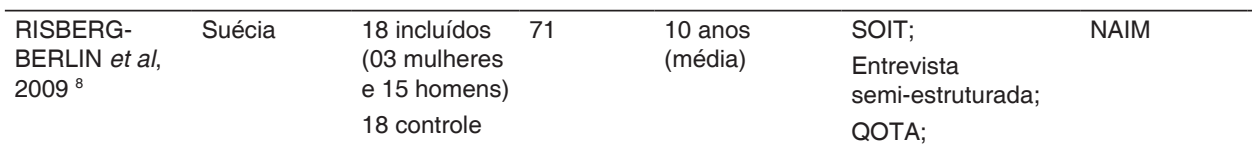

Escore do SOIT à Antes da reabilitação: $61 \%$ anosmia; $39 \%$ detectavam odores; Após 6 meses: $70 \%$ dos pacientes com anosmia passaram a ter hiposmia; Após 36 meses: $22 \%$ anosmia e $78 \%$ normosmia e hiposmia; 12 dos 18 voluntários usuaram o NAIM diariamente;

6 voluntários não usaram (2 normosmia/hiposmia; 4 anosmia);

\begin{tabular}{|c|c|c|c|c|c|}
\hline $\begin{array}{l}\text { GOEKTAS et } \\
a l, 2008^{9}\end{array}$ & Alemanha & $\begin{array}{l}16(03 \\
\text { mulheres e } \\
13 \text { homens) }\end{array}$ & 63 & - & $\begin{array}{l}\text { SStb; } \\
\text { Escala subjetiva de } \\
10 \text { pontos; }\end{array}$ \\
\hline $\begin{array}{l}\text { GOKTAS et al, } \\
2008^{5}\end{array}$ & Alemanha & $\begin{array}{l}16(02 \\
\text { mulheres e } \\
14 \text { homens) }\end{array}$ & 62 & $\begin{array}{l}\text { Entre } 1995 \text { e } \\
2003\end{array}$ & $\begin{array}{l}\text { SStb; } \\
\text { Escala subjetiva de } \\
10 \text { pontos; }\end{array}$ \\
\hline
\end{tabular}

Melhora maior na função olfativa após o uso da SV (75\%) em comparação com a LB; SV de mais fácil utilização;

Antes da técnica todos tinha anosmia (3 pontos em média no teste); Após a LB passou para a média de 7 pontos: 5 pacientes com 8 pontos (limite superior da anosmia) e 2 com pontuação superior passando da anosmia para hiposmia;

\begin{tabular}{|c|c|c|c|c|c|c|c|}
\hline $\begin{array}{l}\text { RISBERG- } \\
\text { BERLIN; } \\
\text { MOLLER; } \\
\text { FINIZIA, } 2007^{10}\end{array}$ & Suécia & $\begin{array}{l}24 \text { (03 } \\
\text { mulheres e } \\
21 \text { homens) }\end{array}$ & 68 & 7 anos (média) & $\begin{array}{l}\text { Entrevista } \\
\text { Semi-estruturada; } \\
\text { SOIT; } \\
\text { QOTA; }\end{array}$ & NAIM & $\begin{array}{l}\text { Antes do tratamento à } 10 \text { dos } 24 \\
(42 \%) \text { são capazes de perceber } \\
\text { odores; } 14(58 \%) \text { tem anosmia; } \\
\text { Após } 6 \text { meses de tratamento } \\
\text { à } 87 \%(n=20) \text { foram capazes } \\
\text { de perceber odores; Após } 12 \\
\text { meses de tratamento à } 88 \% \\
(n=21) \text { percebem odores;Com } \\
\text { a reabilitação } 63 \%(n=15) \text { foram } \\
\text { classificados como normosmia no } \\
\text { final do estudo. }\end{array}$ \\
\hline
\end{tabular}




\begin{tabular}{|c|c|c|c|c|c|c|c|}
\hline $\begin{array}{l}\text { RISBERG- } \\
\text { BERLIN; } \\
\text { YLITALO; } \\
\text { FINIZIA, 2006 }{ }^{11}\end{array}$ & Suécia & $\begin{array}{l}24(03 \\
\text { mulheres e } \\
21 \text { homens) }\end{array}$ & 68 & $\begin{array}{l}\text { Pelo menos } 5 \\
\text { meses }\end{array}$ & $\begin{array}{l}\text { Entrevista } \\
\text { Semi-estruturada; } \\
\text { SOIT; } \\
\text { QOTA; }\end{array}$ & NAIM & $\begin{array}{l}\text { Antes do tratamento à } 42 \% \\
\text { (normosmia [n=6]; hiposmia } \\
\text { [n=4]) são capazes de perceber } \\
\text { odores; } 58 \% \text { ( } n=14) \text { tem anosmia; } \\
13(72 \%) \text { dos } 18 \text { pacientes com } \\
\text { alteração no olfato que utilizaram } \\
\text { o NAIM apresentaram melhora; } \\
\text { Dos } 14 \text { com anosmia } 7 \text { passaram } \\
\text { a perceber odores após uma } \\
\text { sessão da intervenção; }\end{array}$ \\
\hline $\begin{array}{l}\text { HILGERS et } \\
a L, 2002^{12}\end{array}$ & Holanda & $\begin{array}{l}41(10 \\
\text { mulheres e } \\
31 \text { homens) }\end{array}$ & 63 & 6,5 anos & $\begin{array}{l}\text { ODT [POPS]; } \\
\text { SDT; }\end{array}$ & NAIM & $\begin{array}{l}46 \%(n=19) \text { foram considerados } \\
\text { com normosmia; Houve } \\
\text { significância com o uso correto } \\
\text { do NAIM ; }\end{array}$ \\
\hline $\begin{array}{l}\text { HILGERS et } \\
a L, 2000^{13}\end{array}$ & Holanda & $\begin{array}{l}44(10 \\
\text { mulheres e } \\
34 \text { homens) }\end{array}$ & 64 & 6 anos & $\begin{array}{l}\text { ODT; } \\
\text { QOTA [POPS]; }\end{array}$ & NAIM & $\begin{array}{l}\text { Antes do tratamento: } 33 \text { anosmia } \\
\text { e } 11 \text { hiposmia/normosmia; } \\
\text { Após o tratamento: } 19 \text { anosmia e } \\
25 \text { hiposmia/normosmia (taxa de } \\
\text { sucesso de } 46 \% \text { ) }\end{array}$ \\
\hline $\begin{array}{l}\text { ARMENGOT } \\
\text { et } a L, 1990^{19}\end{array}$ & Espanha & 20 homens & $\begin{array}{l}\text { Entre } 43 \text { a } \\
75 \text { anos }\end{array}$ & $\begin{array}{l}9 \text { meses a } 20 \\
\text { anos }\end{array}$ & Método de Elsberg & $\begin{array}{l}\text { Prótese } \\
\text { fonatória }\end{array}$ & $\begin{array}{l}\text { Sem prótese: maior limiar de } \\
\text { percepção para os estímulos } \\
\text { olfativos puros; } \\
\text { Com prótese: Limiar olfativo puro } \\
\text { permanece dentro dos valores } \\
\text { normais; }\end{array}$ \\
\hline $\begin{array}{l}\text { SCHWARTZ } \\
\text { et } a L, 1987^{7}\end{array}$ & USA & $\begin{array}{l}\text { i } 12 \text { ( } 2 \\
\text { mulheres e } \\
10 \text { homens); } \\
\text { ii } 30 \text { ( } 6 \\
\text { mulheres e } \\
24 \text { homens) } \\
25 \text { controle }\end{array}$ & $\begin{array}{l}61,6 \mathrm{i} \\
63 \mathrm{ii}\end{array}$ & $\begin{array}{l}2 \text { meses a } 20 \\
\text { anos }\end{array}$ & $\begin{array}{l}\text { Teste de limiar } \\
\text { de detecção de } \\
\text { odores i; } \\
\text { Teste de } \\
\text { identificação } \\
\text { de odores com } \\
\text { "odorant confusion } \\
\text { matrix" ii; }\end{array}$ & LB ii & $\begin{array}{l}\text { Reversão da hiposmia por } \\
\text { estímulos do nervo trigêmio; } \\
\text { Reversão da hiposmia não é } \\
\text { completa se não estimular o nervo } \\
\text { olfativo; O teste de identificação } \\
\text { com a matriz foi dependente da } \\
\text { taxa de fluxo inspiratório com e } \\
\text { sem a LB. }\end{array}$ \\
\hline
\end{tabular}

SStb = Sniffin Sticks test battery; SOIT =The Scandinavian Odor Identification Test ; QOTA = Questions on Odor, Taste and Appetite; POPS =Present Odor Perception Scale; ODT= Odor Detection Test; SDT = Smell Disk Test; LB = Larynx bypass; SV = Scent-diffusing ventilator; NAIM: Nasal Airflow-Inducing Maneuver; i = (grupo 1) estudo inicial; ii= (grupo 2) 30 sujeitos (10 do grupo 1)

$\mathrm{Na}$ análise da função gustativa observou-se que os estudos utilizaram questionários e entrevistas semi-estruturadas, com abordagem subjetiva e com proposta de autoavaliação 4, 8, 10, 11, 13. Entende-se que as alterações do paladar podem ser consideradas como consequência da diminuição da função olfativa, o que pode justificar a falta de análises com testes padronizados para a função gustativa.

Observa-se que até mesmo em artigos que propõem apenas a avaliação da função gustativa são utilizados testes subjetivos, principalmente de soluções aquosas, com instrumentos não padronizados ${ }^{18}$, o que demonstra a carência de estudos mais objetivos para análise desta função.

As técnicas de intervenção encontradas nos artigos selecionados para esta revisão descrevem principalmente o uso de dispositivos para restabelecer o fluxo aéreo nasal, reconectando a via aérea superior à inferior, como também técnicas para trabalhar a musculatura orofacial a fim de recuperar esse fluxo aéreo. Porém em um único estudo foi analisada a possibilidade de utilização da prótese fonatória como técnica de intervenção, sendo atestada pouca eficiência neste método ${ }^{19}$.

Alguns estudos têm demonstrado a eficácia na utilização do "Larynx Bypass" (LB), um tubo flexível com o propósito de conectar a boca do paciente ao traqueostoma e restabelecer o fluxo aéreo, ocasionando melhora olfativa a partir da circulação do ar na passagem nasal, necessitando que o paciente relaxe o palato para que não obstrua a nasofaringe $5,7,9$

Pesquisa realizada na Alemanha discute a praticidade desse método e propõe a técnica do Scentdiffusing ventilator (SV), em que um pequeno ventilador é acoplado a uma máscara, transportando o ar diretamente ao nariz, proporcionando a entrada de moléculas odoríferas de forma passiva à cavidade nasal ${ }^{9}$.

Em ambas as técnicas há evidências na melhoria da olfação, porém constata-se uma melhora maior através do uso do SV em comparação com o uso da LB, além de ser de mais fácil utilização. Em contrapartida, é discutível o custo desse recurso, em que há necessidade de maior tecnologia, já que no LB utiliza-se apenas um tubo flexível, o que sugere menor aporte financeiro.

Já o Nasal Airflow-Inducing Maneuver (NAIM) é a técnica mais comumente utilizada em estudos que propõem reabilitação das funções olfativas e gustativas ${ }^{3,4,8,10-13}$. Esse dispositivo tem como proposta criar uma pressão negativa na cavidade oral e orofaríngea para induzir o fluxo aéreo nasal, permitindo a chegada de moléculas odoríferas novamente ao 
neuroepitélio olfativo. Esta técnica conhecida como polite yawning, consiste num "bocejo" prolongado com o movimento simultâneo de retração da mandíbula, do assoalho da boca, da língua, da base da língua e do palato mole, mantendo os lábios firmemente fechados ${ }^{13}$.

Observa-se que esta técnica tem se mostrado eficaz no restabelecimento da função olfativa, convertendo indivíduos com anosmia a retomarem a capacidade de perceber odores, tanto a curto prazo como a após períodos prolongados de uso, além de ser de fácil aprendizagem pelos usuários.

Em pesquisa realizada na Suécia com 24 indivíduos foi utilizada o NAIM como método de reabilitação, constatando-se que metade dos pacientes que apresentavam anosmia passaram a perceber odores após ser administrada uma única sessão dessa técnica ${ }^{11}$.

Este fato reforça a importância do investimento em propostas de reabilitação, já que é possível detectar evoluções em um curto período de tempo, repercutindo em melhoria na função olfativa e na qualidade de vida desses sujeitos. Esses resultados podem servir de estímulos para pesquisadores investirem nesta área e proporem novas formas de intervenção na prática clínica.

Nos estudos selecionados percebe-se que a intenção de pesquisar propostas de reabilitação nem sempre repercute em investimentos para prática clínica, e embora haja a preocupação em incentivar essa prática ${ }^{4}$ apenas um estudo expõe a análise de intervenções na clínica e na assistência domiciliar ${ }^{3}$. Diante disso, sugerem-se novos estudos que relatem a aplicabilidade dessas técnicas no cotidiano de profissionais que prestam assistência a essa população.

Observa-se ainda que as diversas técnicas existentes para reativação do olfato não propõem intervir diretamente na função gustativa. A ausência de métodos especificos para reabilitar a função do paladar, tal como a inexistência de testes padronizados para avaliar essa função, reitera a hipótese de que as alterações olfativas em laringectomizados total podem repercutir em distúrbios gustativos e que a reativação da função olfativa pode também melhorar o paladar nessa população.

\section{CONCLUSÃO}

Nesta revisão os testes utilizados para avaliação da função olfativa em sua maioria preocuparamse em fazer uma análise quantitativa com uso de instrumentos padronizados, o que sugere uma preocupação com os resultados apresentados, já que tem como finalidade comprovar a eficácia de uma técnica de intervenção, embora para avaliação do paladar inexistam testes e métodos específicos de reabilitação

Todos os estudos demonstraram eficácia na utilização de técnicas de reabilitação para a função do olfato em laringectomizados total. Das várias técnicas existentes a que vem sendo mais comumente utilizada é Nasal Airflow-Inducing Maneuver (NAIM), sendo um método de aprendizagem rápida, com resultados evidentes na reativação do olfato, podendo apresentar efeitos imediatos após administração.

Observa-se maior preocupação na reabilitação da função olfativa em detrimento do paladar, considerando-se que as alterações do paladar em laringectomizados totais sejam conseqüência das modificações no olfato, sugerindo que a reativação do olfato repercutirá também em melhorias na função gustativa.

\section{ABSTRACT}

Background: in total laryngectomy there occurs the transfer of the nasal airflow definitely to the tracheostoma, which could cause a decrease in the perception of smell and taste. There have been recently developed methods of intervention in order to improve performance in laryngectomized smell and taste, although few studies have investigated this issue. Purpose: this study aimed to systematically review the evidence of how the techniques involved in the rehabilitation of the functions of smell and taste have an effect on individuals with total laryngectomy. Conclusion: in this review all the selected studies demonstrated effective use of rehabilitation techniques for the role of olfaction in laryngectomized. As for taste, there were no proposals in order to intervene directly in this function.

KEYWORDS: Laryngectomy; Smell; Taste; Olfaction Disorders; Taste Disorders; Rehabilitation 


\section{REFERÊNCIAS}

1. Hannickel S, Zago MMF, Barbeira CBS, Sawada NO. O comportamento dos laringectomizados frente à imagem corporal. Revista Brasileira de Cancerologia. 2002 jul-ago-set; 48(3): 333-9.

2. Welge-luessen A, Kobal G, Wolfensberger M. Assessing olfactory function in laryngectomees using the Sniffin.sticks test battery and chemosensory evoked potentials. Laryngoscope. 2000; 110: 303-7. 3. Ward E, Coleman A, van As-Brooks C, Kerle S. Rehabilitation of olfaction post-laryngectomy:a randomized control trial comparing clinician assisted versus a home practice approach. Clin Otolaryngol. 2010 fev; 35(1):39-45.

4. Morales-puebla JM, Morales-Puebla AF, JiménezAntolín JA, Muñoz-Platón E, Padilla-Parrado $M$, Chacón-Martínez J. Olfactory rehabilitation after total laryngectomy. Acata Otorrinolaringol Esp. 2010 mar-abr; 61(2):128-34.

5. Goktas O, Fleiner F, Paschen C, Lammert I, Schrom T. Rehabilitation of the olfactory sense after laryngectomy: long-term use of the larynx bypass. Ear Nose Throat J. 2008 Set;87(9): 528-30.

6. Marco J, Gimenez JA, Morera H. Olfaction in laryngectomy. Rev Esp Otoneurooftalmol Neurocir. 1954 Nov-Dez; 13(76):380-94.

7. Schwartz DN, Mozell MM, Leopold DL, Youngentob SL, Sheehe PR. Improvement of olfaction in laryngectomized patients with the larynx bypass. Laryngoscope.1987 Nov; 97(11): 1280-6.

8. Risberg-Berlin B, Rydén A, Möller RY, Finizia C. Effects of total laryngectomy on olfactory function, health-related quality of life, and communication: a 3-year follow-up study. BMC Ear, Nose and Throat Disorders.[periódico na internet] 2009[acesso em 2010 out. 19]; 9 (8):[ 9 p.]. Disponível em: http:// www.biomedcentral.com/1472-6815/9/8.

9. Goektas O, Fleiner F, Frieler K, Scherer H, Paschen C. The scent-diffusing ventilator for rehabilitation of olfactory function after laryngectomy. Am $\mathrm{J}$ Rhinol. 2008 Set-Out;22(5):487-90.

10. Risberg-Berlin B, Moller RY, Finizia C. Effectiveness of Olfactory Rehabilitation With the Nasal Airflow-Inducing Maneuver After Total Laryngectomy: One-Year Follow-up Study. Arch otolaryngol head neck surg. 2007 Jul.; 133 (7):650-4. 11. Risberg-Berlin B, Ylitalo R, Finizia C. Screening and rehabilitation of olfaction after total laryngectomy in swedish patients: results from an intervention study using the nasal airflow-inducing maneuver. Arch Otolaryngol. Head Neck Surg. 2006 mar.; 132: 301-6.

12. Hilgers FJM, Jansen HA, Van As CJ, Polak MF, Muller MJ, Van Dam FSAM. Long-term results of olfaction rehabilitation using the nasal airflowinducing ("polite Yawning") maneuver after total laryngectomy. Arch Otolaryngol. Head Neck Surg. 2002 jul.; 128: 648 -54.

13. Hilgers FJM, Van Dam FSAM, Keyzers S, Koster MN, Van As CJ, Muller MJ. Rehabilitation of olfaction after laryngectomy by means of a nasal airflow-inducing maneuver: The "polite yawning" technique. Arch Otolaryngol. Head Neck Surg. 2000 jun.; 126: 726 -32.

14. Oliveira IB de, Costa CC, Chagas JFS, Rochetti ECG, Oliveira LO de. Comunicação oral de laringectomizados com prótese traqueoesofágica: análise comparativa pré e pós-treino. Pró-Fono R. Atual. Cient. 2005 Aug.; 17(2): 165-74.

15. Paula FC, Gama RR. Avaliação de qualidade de vida em laringectomizados totais. Rev bras cir. cabeça pescoço. 2009 Jul-Set; 38(3):177-82.

16. Cleto ML, Pedalini LM, Junior JFM. Reativação do olfato em laringectomizados totais. Arquivos internacionais de otorrinolaringologia. 2005 Abr-Jun; 9(2):102-7.

17. Wünsch Filho I. The epidemiology of laryngeal cancer in Brazil. Sao Paulo Med J. 2004;122(5):188-94.

18. Kashima HK, Kalinowski B. Taste impairment following laryngectomy. Ear Nose Throat J. 1979 Fev;58(2):88-92.

19. Armengot M, Algarra JM, Franco MR, Gimenez $F$. Olfaccion en los laringectomizados: influencia de la protesis fonatoria. Anales O.R.L. iber.-Amer. 1990; 17: 99-105.
http://dx.doi.org/10.1590/S1516-18462011005000100

RECEBIDO EM: 07/01/2011

ACEITO EM: 27/04/2011

Endereço para correspondência:

Ada Salvetti Cavalcanti Caldas

Rua Guedes Pereira, 180 - apto. 903 Parnamirim

Recife - Pernambuco

CEP: 52060-150

E-mail: adasc@ hotmail.com 